\title{
PARTIAL SUMS FOR A CERTAIN SUBCLASS OF MEROMORPHIC UNIVALENT FUNCTIONS
}

\author{
M. K. AOUF, A. O. MOSTAFA, A. Y. LASHIN AND B. M. MUNASSAR
}

\begin{abstract}
In this paper, the class $\Sigma_{\lambda}(\alpha, \beta, \gamma)$ of univalent meromorphic functions defined using the Ruscheweyh derivative in the punctured unit disk $\mathrm{U}^{*}$ is introduced. We study some results concerning the partial sums of meromorphic univalent starlike functions and meromorphic univalent convex functions.
\end{abstract}

\section{INTRODUCTION}

Let $\Sigma$ denote the class of meromorphic functions of the form:

$$
f(z)=\frac{1}{z}+\sum_{k=1}^{\infty} a_{k} z^{k} \quad\left(a_{k} \geq 0\right)
$$

which are regular and univalent in the punctured unit disc $U^{*}=\{z: z \in \mathbb{C}$ and $0<|z|<1\}=U \backslash\{0\}$. Let $g \in \Sigma$, be given by

$$
g(z)=\frac{1}{z}+\sum_{k=1}^{\infty} b_{k} z^{k}
$$

then the Hadamard product (or convolution) of $f$ and $g$ is given by

$$
(f * g)(z)=\frac{1}{z}+\sum_{k=1}^{\infty} a_{k} b_{k} z^{k}=(g * f)(z) .
$$

A function $f \in \Sigma$ is said to be meromorphically starlike of order $\alpha$ if

$$
\operatorname{Re}\left\{-\frac{z f^{\prime}(z)}{f(z)}\right\}>\alpha \quad(z \in U ; 0 \leq \alpha<1) .
$$

2010 Mathematics Subject Classification. 30C45.

Key words and phrases. Partial sums, meromorphic univalent functions, Ruscheweyh derivative. 
Denote by $\Sigma^{*}(\alpha)$ the class of all meromorphically starlike functions of order $\alpha$. A function $f \in \Sigma$ is said to be meromorphically convex of order $\alpha$ if

$$
\operatorname{Re}\left\{-\left(1+\frac{z f^{\prime \prime}(z)}{f^{\prime}(z)}\right)\right\}>\alpha(z \in U ;(0 \leq \alpha<1)) .
$$

Denote by $\Sigma_{k}^{*}(\alpha)$ the class of all meromorphically convex functions of order $\alpha$. We note that

$$
f(z) \in \Sigma_{k}^{*}(\alpha) \Longleftrightarrow-z f^{\prime}(z) \in \Sigma^{*}(\alpha) .
$$

The classes $\Sigma^{*}(\alpha)$ and $\Sigma_{k}^{*}(\alpha)$ had been extensively studied by Pommerenke [7], Miller [6] and others.

For $\lambda>-1$, the Ruscheweyh derivative of order $\lambda$ is denoted by $D^{\lambda} f$ and is defined for function of the form (1.1) as follows: If

$$
f(z)=\frac{1}{z}+\sum_{k=1}^{\infty} a_{k} z^{k}
$$

then

$$
D^{\lambda} f(z)=\frac{1}{z(1-z)^{\lambda+1}} * f(z)=z^{-1}+\sum_{k=1}^{\infty} D_{k}(\lambda) a_{k} z^{k}, \quad z \in U^{*},
$$

where

$$
D_{k}(\lambda)=\frac{(\lambda+1)(\lambda+2) \ldots(\lambda+k+1)}{(k+1) !} .
$$

For $\beta \geq 0,0 \leq \alpha<1,0 \leq \gamma<\frac{1}{2}$ and $\lambda>-1$, Atshan and Kulkarni [4] and Atshan [3] defined the class $\Sigma_{\lambda}(\alpha, \beta, \gamma)$ consisting of functions of the form (1.1) and satisfying the analytic criterion:

$$
\begin{aligned}
& -\operatorname{Re}\left\{\frac{z\left(D^{\lambda} f(z)\right)^{\prime}+\gamma z^{2}\left(D^{\lambda} f(z)\right)^{\prime \prime}}{(1-\gamma) D^{\lambda} f(z)+\gamma z\left(D^{\lambda} f(z)\right)^{\prime}}+\alpha\right\} \geq \\
& \beta\left|\frac{z\left(D^{\lambda} f(z)\right)^{\prime}+\gamma z^{2}\left(D^{\lambda} f(z)\right)^{\prime \prime}}{(1-\gamma) D^{\lambda} f(z)+\gamma z\left(D^{\lambda} f(z)\right)^{\prime}}+1\right|(z \in U) .
\end{aligned}
$$

We note that:

$\Sigma_{0}(\alpha, 0,0)=\Sigma^{*}(\alpha)(0 \leq \alpha<1)$ (see Pommerenke [7]).

Also, we note that

$$
\begin{aligned}
& \Sigma_{\lambda}(\alpha, \beta, 0)=\Sigma_{\lambda}^{*}(\alpha, \beta)= \\
& \quad-\operatorname{Re}\left\{\frac{z\left(D^{\lambda} f(z)\right)^{\prime}}{D^{\lambda} f(z)}+\alpha\right\} \geq \beta\left|\frac{z\left(D^{\lambda} f(z)\right)^{\prime}}{D^{\lambda} f(z)}+1\right|(z \in U) .
\end{aligned}
$$

For $\beta \geq 0,0 \leq \alpha<1$, and $\lambda>-1$, we denote by $\Sigma_{k, \lambda}^{*}(\alpha, \beta)$ the subclass of $\Sigma$ consisting of functions of the form (1.1) and satisfying the analytic 
criterion:

$$
-\operatorname{Re}\left\{1+\frac{z\left(D^{\lambda} f(z)\right)^{\prime \prime}}{D^{\lambda} f^{\prime}(z)}+\alpha\right\} \geq \beta\left|2+\frac{z\left(D^{\lambda} f(z)\right)^{\prime \prime}}{D^{\lambda} f^{\prime}(z)}\right|(z \in U),
$$

We note that:

$\Sigma_{k, 0}(\alpha, 0)=\Sigma_{k}^{*}(\alpha)(0 \leq \alpha<1)$ (see Pommerenke [7]).

It is easy to observe from (1.9) and (1.10) that

$$
f(z) \in \Sigma_{k, \lambda}^{*}(\beta, \alpha) \Longleftrightarrow-z f^{\prime}(z) \in \Sigma_{\lambda}^{*}(\beta, \alpha) .
$$

In order to prove our results for functions belonging to the class $\Sigma_{\lambda}(\alpha, \beta, \gamma)$ we shall need the following lemma given by Atshan and Kulkarni [4] .

Lemma 1. [4, Theorem 2.1] Let the function $f$ be defined by (1.1). Then $f \in \Sigma_{\lambda}(\alpha, \beta, \gamma)$ if and only if

$$
\sum_{k=1}^{\infty}(1+\gamma k-\gamma)[k(1+\beta)+(\beta+\alpha)] D_{k}(\lambda) a_{k} \leq(1-\alpha)(1-2 \gamma)
$$

where $0 \leq \alpha<1, \beta \geq 0,0 \leq \gamma<\frac{1}{2}, \lambda>-1$, and $D_{k}(\lambda)$ is given by (1.7).

Taking $\gamma=0$ in Lemma 1, we obtain the following corollary.

Corollary 1. Let the function $f$ defined by (1.1). Then $f \in \Sigma_{\lambda}^{*}(\beta, \alpha)$ if and only if

$$
\sum_{k=1}^{\infty}[k(1+\beta)+(\beta+\alpha)] D_{k}(\lambda) a_{k} \leq(1-\alpha) .
$$

By using Corollary 1 and (1.11), we can prove the following lemma.

Lemma 2. Let the function $f$ defined by (1.1). Then $f \in \Sigma_{k, \lambda}^{*}(\beta, \alpha)$ if and only if

$$
\sum_{k=1}^{\infty} k[k(1+\beta)+(\beta+\alpha)] D_{k}(\lambda) a_{k} \leq(1-\alpha) .
$$

In this paper, applying the technique used by Silverman [8], we will investigate the ratio of a function of the form (1.1) to its sequence of partial sums $f_{n}(z)=\frac{1}{z}+\sum_{k=1}^{n} a_{k} z^{k}$ when the coefficients of $f$ are sufficiently small to satisfy condition (1.13) or (1.14). More precisely, we will determine sharp lower bounds for

$$
\operatorname{Re}\left\{\frac{f(z)}{f_{n}(z)}\right\}, \operatorname{Re}\left\{\frac{f_{n}(z)}{f(z)}\right\}, \operatorname{Re}\left\{\frac{f^{\prime}(z)}{f_{n}^{\prime}(z)}\right\} \text { and } \operatorname{Re}\left\{\frac{f_{n}^{\prime}(z)}{f^{\prime}(z)}\right\} .
$$

In the sequel, we will make use of well-known result that $\operatorname{Re}\left\{\frac{1+w(z)}{1-w(z)}\right\}>0$ $(z \in U)$ if and only if $w(z)=\sum_{k=1}^{\infty} c_{k} z^{k}$ satisfies the inequality $|w(z)| \leq|z|$. 
Unless otherwise stated, we will assume that $f$ is of the form (1.1) and its sequence of partial sums is denoted by $f_{n}(z)=\frac{1}{z}+\sum_{k=1}^{n} a_{k} z^{k}$.

For the notational convenience we shall henceforth denote

$$
\delta_{k}(\lambda, \beta, \alpha)=[k(1+\beta)+(\beta+\alpha)] D_{k}(\lambda) .
$$

\section{MAin RESUltS}

Theorem 1. If $f$ of the form (1.1) satisfies condition (1.13), then

$$
\operatorname{Re}\left\{\frac{f(z)}{f_{n}(z)}\right\} \geq \frac{\delta_{n+1}(\lambda, \beta, \alpha)-(1-\alpha)}{\delta_{n+1}(\lambda, \beta, \alpha)} \quad(z \in U) .
$$

The result is sharp, with extremal function

$$
f(z)=\frac{1}{z}+\frac{1-\alpha}{\delta_{n+1}(\lambda, \beta, \alpha)} z^{n+1} \quad(n \geq 1) .
$$

Proof. We may write

$$
\begin{aligned}
\frac{\delta_{n+1}(\lambda, \beta, \alpha)}{1-\alpha} & {\left[\frac{f(z)}{f_{n}(z)}-\frac{\delta_{n+1}(\lambda, \beta, \alpha)-(1-\alpha)}{\delta_{n+1}(\lambda, \beta, \alpha)}\right] } \\
& =\frac{1+\sum_{k=1}^{n} a_{k} z^{k+1}+\frac{\delta_{n+1}(\lambda, \beta, \alpha)}{1-\alpha} \sum_{k=n+1}^{\infty} a_{k} z^{k+1}}{1+\sum_{k=1}^{n} a_{k} z^{k+1}} \\
& =\frac{1+A(z)}{1+B(z)} .
\end{aligned}
$$

Set $\frac{1+A(z)}{1+B(z)}=\frac{1+w(z)}{1-w(z)}$, so that $w(z)=\frac{A(z)-B(z)}{2+A(z)+B(z)}$. Then

$$
w(z)=\frac{\frac{\delta_{n+1}(\lambda, \beta, \alpha)}{1-\alpha} \sum_{k=n+1}^{\infty} a_{k} z^{k+1}}{2+2 \sum_{k=1}^{n} a_{k} z^{k+1}+\frac{\delta_{n+1}(\lambda, \beta, \alpha)}{1-\alpha} \sum_{k=n+1}^{\infty} a_{k} z^{k+1}}
$$

and

$$
|w(z)| \leq \frac{\frac{\delta_{n+1}(\lambda, \beta, \alpha)}{1-\alpha} \sum_{k=n+1}^{\infty} a_{k}}{2-2 \sum_{k=1}^{n} a_{k}-\frac{\delta_{n+1}(\lambda, \beta, \alpha)}{1-\alpha} \sum_{k=n+1}^{\infty} a_{k}} .
$$

Now $|w(z)| \leq 1$ if and only if

$$
2\left(\frac{\delta_{n+1}(\lambda, \beta, \alpha)}{1-\alpha}\right) \sum_{k=n+1}^{\infty} a_{k} \leq 2-2 \sum_{k=1}^{n} a_{k},
$$


which is equivalent to

$$
\sum_{k=1}^{n} a_{k}+\left(\frac{\delta_{n+1}(\lambda, \beta, \alpha)}{1-\alpha}\right) \sum_{k=n+1}^{\infty} a_{k} \leq 1 .
$$

It suffices to show that the left hand side of (2.3) is bounded above by $\sum_{k=1}^{\infty}\left(\frac{\delta_{k}(\lambda, \beta, \alpha)}{1-\alpha}\right) a_{k}$, which is equivalent to

$$
\sum_{k=1}^{n} \frac{\delta_{k}(\lambda, \beta, \alpha)-(1-\alpha)}{1-\alpha} a_{k}+\sum_{k=n+1}^{\infty} \frac{\delta_{k}(\lambda, \beta, \alpha)-\delta_{n+1}(\lambda, \beta, \alpha)}{1-\alpha} a_{k} \geq 0 .
$$

To see that the function $f$ given by (2.2) gives the sharp result, we observe for $z=r e^{\pi i /(n+2)}$ that

$$
\begin{aligned}
\frac{f(z)}{f_{n}(z)} & =1+\frac{1-\alpha}{\delta_{n+1}(\lambda, \beta, \alpha)} z^{n+2} \\
& \rightarrow 1-\frac{1-\alpha}{\delta_{n+1}(\lambda, \beta, \alpha)} \\
& =\frac{\delta_{n+1}(\lambda, \beta, \alpha)-(1-\alpha)}{\delta_{n+1}(\lambda, \beta, \alpha)} \text { when } r \rightarrow 1^{-} .
\end{aligned}
$$

Therefore the proof of Theorem 1 is completed.

Theorem 2. If $f$ of the form (1.1) satisfies condition (1.14), then

$$
\operatorname{Re}\left\{\frac{f(z)}{f_{n}(z)}\right\} \geq \frac{(n+1) \delta_{n+1}(\lambda, \beta, \alpha)-(1-\alpha)}{(n+1) \delta_{n+1}(\lambda, \beta, \alpha)} \quad(z \in U) .
$$

The result is sharp for every $n$, with extremal function

$$
f(z)=\frac{1}{z}+\frac{1-\alpha}{(n+1) \delta_{n+1}(\lambda, \beta, \alpha)} z^{n+1} \quad(n \geq 1) .
$$

Proof. We may write

$$
\begin{gathered}
\frac{(n+1) \delta_{n+1}(\lambda, \beta, \alpha)}{1-\alpha}\left[\frac{f(z)}{f_{n}(z)}-\frac{(n+1) \delta_{n+1}(\lambda, \beta, \alpha)-(1-\alpha)}{(n+1) \delta_{n+1}(\lambda, \beta, \alpha)}\right] \\
=\frac{1+\sum_{k=1}^{n} a_{k} z^{k+1}+\frac{(n+1) \delta_{n+1}(\lambda, \beta, \alpha)}{1-\alpha} \sum_{k=n+1}^{\infty} a_{k} z^{k+1}}{1+\sum_{k=1}^{n} a_{k} z^{k+1}} \\
=\frac{1+w(z)}{1-w(z)}
\end{gathered}
$$


where

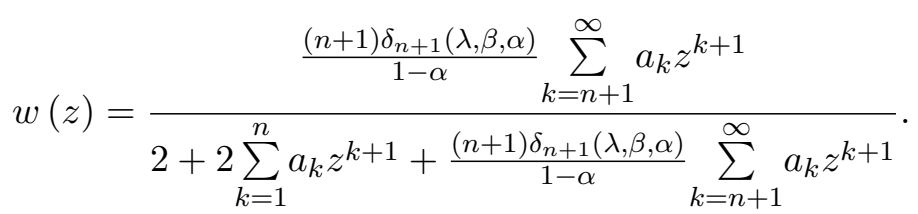

Now

$$
|w(z)| \leq \frac{\frac{(n+1) \delta_{n+1}(\lambda, \beta, \alpha)}{1-\alpha} \sum_{k=n+1}^{\infty} a_{k}}{2-2 \sum_{k=1}^{n} a_{k}-\frac{(n+1) \delta_{n+1}(\lambda, \beta, \alpha)}{1-\alpha} \sum_{k=n+1}^{\infty} a_{k}} .
$$

if

$$
\sum_{k=1}^{n} a_{k}+\left(\frac{(n+1) \delta_{n+1}(\lambda, \beta, \alpha)}{1-\alpha}\right) \sum_{k=n+1}^{\infty} a_{k} \leq 1 .
$$

The left hand side of (2.6) is bounded above by

$$
\sum_{k=1}^{\infty}\left(\frac{k \delta_{k}(\lambda, \beta, \alpha)}{1-\alpha}\right) a_{k}
$$

if

$$
\begin{aligned}
\frac{1}{1-\alpha}\left\{\sum_{k=1}^{n}\left[k \delta_{k}(\lambda, \beta, \alpha)-(1-\alpha)\right] a_{k}\right. \\
\\
\left.\quad+\sum_{k=n+1}^{\infty}\left[k \delta_{k}(\lambda, \beta, \alpha)-(n+1) \delta_{n+1}(\lambda, \beta, \alpha)\right] a_{k}\right\} \geq 0
\end{aligned}
$$

and the proof of Theorem 2 is completed.

We next determine bounds for $\operatorname{Re}\left\{\frac{f_{n}(z)}{f(z)}\right\}$.

Theorem 3. (a) If $f$ of the form (1.1) satisfies condition (1.13), then

$$
\operatorname{Re}\left\{\frac{f_{n}(z)}{f(z)}\right\} \geq \frac{\delta_{n+1}(\lambda, \beta, \alpha)}{(1-\alpha)+\delta_{n+1}(\lambda, \beta, \alpha)} \quad(z \in U) .
$$

(b) If $f$ of the form (1.1) satisfies condition (1.14), then

$$
\operatorname{Re}\left\{\frac{f_{n}(z)}{f(z)}\right\} \geq \frac{(n+1) \delta_{n+1}(\lambda, \beta, \alpha)}{(1-\alpha)-(n+1) \delta_{n+1}(\lambda, \beta, \alpha)} \quad(z \in U) .
$$

Equalities hold in (a) and (b) for the functions given by (2.2) and (2.5), respectively. 
Proof. We prove $(a)$. The proof of $(b)$ is similar to $(a)$ and will be omitted. We write

$$
\begin{gathered}
\frac{(1-\alpha)+\delta_{n+1}(\lambda, \beta, \alpha)}{1-\alpha}\left[\frac{f_{n}(z)}{f(z)}-\frac{\delta_{n+1}(\lambda, \beta, \alpha)}{(1-\alpha)+\delta_{n+1}(\lambda, \beta, \alpha)}\right] \\
=\frac{1+\sum_{k=1}^{n} a_{k} z^{k+1}-\frac{\delta_{n+1}(\lambda, \beta, \alpha)}{1-\alpha} \sum_{k=n+1}^{\infty} a_{k} z^{k+1}}{1+\sum_{k=1}^{\infty} a_{k} z^{k+1}} \\
=\frac{1+w(z)}{1-w(z)},
\end{gathered}
$$

where

$$
|w(z)| \leq \frac{\frac{(1-\alpha)+\delta_{n+1}(\lambda, \beta, \alpha)}{1-\alpha} \sum_{k=n+1}^{\infty} a_{k}}{2-2 \sum_{k=1}^{n} a_{k}-\frac{\delta_{n+1}(\lambda, \beta, \alpha)-(1-\alpha)}{1-\alpha} \sum_{k=n+1}^{\infty} a_{k}} \leq 1 .
$$

This last inequality is equivalent to

$$
\sum_{k=1}^{n} a_{k}+\left(\frac{\delta_{n+1}(\lambda, \beta, \alpha)}{1-\alpha}\right) \sum_{k=n+1}^{\infty} a_{k} \leq 1 .
$$

Since the left hand side of (2.9) is bounded above by

$$
\sum_{k=1}^{\infty}\left(\frac{\delta_{k}(\lambda, \beta, \alpha)}{1-\alpha}\right) a_{k}
$$

the proof is completed.

We next turn to ratios involving derivatives. The proof of Theorem 4 below follows the pattern of those in Theorem 1 and $(a)$ of Theorem 3 and so the details may be omitted.

Theorem 4. If $f$ of the form (1.1) satisfies condition (1.13), then

$$
\begin{aligned}
& \text { (a) } \operatorname{Re}\left\{\frac{f^{\prime}(z)}{f_{n}^{\prime}(z)}\right\} \geq \frac{\delta_{n+1}(\lambda, \beta, \alpha)+(n+1)(1-\alpha)}{\delta_{n+1}(\lambda, \beta, \alpha)} \quad(z \in U), \\
& \text { (b) } \operatorname{Re}\left\{\frac{f_{n}^{\prime}(z)}{f^{\prime}(z)}\right\} \geq \frac{\delta_{n+1}(\lambda, \beta, \alpha)}{\delta_{n+1}(\lambda, \beta, \alpha)-(n+1)(1-\alpha)} \quad(z \in U ; \alpha \neq 0,) .
\end{aligned}
$$

The extremal function for the case $(a)$ is given by (2.2) and the extremal function for the case (b) is given by (2.2) with $\alpha \neq 0$. 
Remark 1. Putting $\beta=0$ and $\lambda=0$ in Theorem 2, we obtain the following corollary:

Corollary 2. If $f$ of the form (1.1) satisfies condition (1.13) (with $\beta=$ 0 and $\lambda=0)$, that is $f \in \Sigma^{*}(\alpha)$, then

$$
\begin{aligned}
& \text { (a) } \operatorname{Re}\left\{\frac{f^{\prime}(z)}{f_{n}^{\prime}(z)}\right\} \geq \frac{2(n+1)-n \alpha}{n+1+\alpha} \quad(z \in U), \\
& \text { (b) } \operatorname{Re}\left\{\frac{f_{n}^{\prime}(z)}{f^{\prime}(z)}\right\} \geq \frac{n+1+\alpha}{\alpha(n+2)} \quad(z \in U ; \alpha \neq 0) .
\end{aligned}
$$

The extremal function for the case ( $a)$ is given by $(2.2)$ (with $\beta=0$ and $\lambda=0$ ) and the extremal function for the case (b) is given by $(2.2)$. (with $\beta=0, \lambda=$ 0 and $\alpha \neq 0$ ).

Remark 2. We note that Corollary 2 corrects the result obtained by Cho and Owa [5, Theorem 4].

Theorem 5. If $f$ of the form (1.1) satisfies condition (1.14), then

$$
\begin{aligned}
& \text { (a) } \operatorname{Re}\left\{\frac{f^{\prime}(z)}{f_{n}^{\prime}(z)}\right\} \geq \frac{\delta_{n+1}(\lambda, \beta, \alpha)-(1-\alpha)}{\delta_{n+1}(\lambda, \beta, \alpha)} \quad(z \in U) . \\
& \text { (b) } \operatorname{Re}\left\{\frac{f_{n}^{\prime}(z)}{f^{\prime}(z)}\right\} \geq \frac{\delta_{n+1}(\lambda, \beta, \alpha)}{(1-\alpha)+\delta_{n+1}(\lambda, \beta, \alpha)} \quad(z \in U) .
\end{aligned}
$$

In both cases, the extremal function is given by (2.5).

Proof. It is well known that $f \in \Sigma_{k}^{*}(\alpha) \Longleftrightarrow-z f^{\prime} \in \Sigma^{*}(\alpha)$. In particular, $f$ satisfies condition (1.14) if and only if $-z f^{\prime}$ satisfies condition (1.13). Thus, $(a)$ is an immediate consequence of Theorem 1 and $(b)$ follows directly from (a) of Theorem 3 .

Remark 3. Puting $\beta=0$ and $\lambda=0$, in the above results, we get the results obtained by Cho and Owa [5] .

Remark 4. Puting $\beta=0$ and $\lambda=0$, in the above results, we get the results obtained by Aouf and Silverman [2 with $p=1]$.

Remark 5. Puting $\beta=0$ and $\lambda=0$, in the above results, we get the results obtained by Aouf and Mostafa [1 with $p=B=1$ and $A=2 \alpha-1,0 \leq \alpha<1$ ]. 


\section{REFERENCES}

[1] M. K. Aouf and A.O.Mostafa, On partial sums of certain meromophic p-valent functions, Math. Comput. Modelling 50 (2009), 1325-1331.

[2] M. K. Aouf and H. Silverman, Partial sums of certain meromophic p-valent functions, J. Inequal. Pure Appl. Math., 7 (4) (2006), Art. 119, 1-7.

[3] W. G. Atshan, Subclass of meromorphic functions with positive coefficients defined by Ruscheweyh derivative II, Surv. Math. Appl., 3 (2008), 67-77.

[4] W. G. Atshan and S. R. Kulkarni, Subclass of meromorphic functions with positive coefficients defined by Ruscheweyh derivative I, J. Rajasthan Acad. Phys. Sci., 69 (2) (2007), 129-140.

[5] N. E. Cho and S. Owa, Partial sums of certain meromorphic functions, J. Ineq. Pure and Appl. Math., 5 (2) (2004), Art. 30.

[6] J. E. Miller, Convex meromorphic mapping and related functions, Proc. Amer. Math. Soc., 25 (1970), 220-228.

[7] Ch. Pommerenke, On meromorphic starlike functions, Pacific J. Math., 13 (1963), 221-235.

[8] H. Silverman, Partial sums of starlike and convex functions, J. Math. Anal. Appl., 209 (1997), 221-227.

(Received: March 13, 2014)

M. K. Aouf, A. O. Mostafa, A. Y. Lashin and B. M. Munassar Department of Mathematics Faculty of Science University of Mansoura Mansoura

Egypt

mkaouf127@yahoo.com adelaeg254@yahoo.com aylashin@yahoo.com bmunassar@yahoo.com 